\title{
A cadaveric microanatomical study of the fascicular topography of the brachial plexus
}

\author{
Sumit Sinha, MCh, ${ }^{1}$ G. Lakshmi Prasad, MCh, ${ }^{1}$ and Sanjeev Lalwani, MD ${ }^{2}$ \\ Departments of ${ }^{1}$ Neurosurgery and ${ }^{2}$ Forensic Medicine, Jai Prakash Narayan Apex Trauma Centre, AllMS, New Delhi, India
}

OBJECTIVE Mapping of the fascicular anatomy of the brachial plexus could provide the nerve surgeon with knowledge of fascicular orientation in spinal nerves of the brachial plexus. This knowledge might improve the surgical outcome of nerve grafting in brachial plexus injuries by anastomosing related fascicles and avoiding possible axonal misrouting. The objective of this study was to map the fascicular topography in the spinal nerves of the brachial plexus.

METHODS The entire right-sided brachial plexus of 25 adult male cadavers was dissected, including all 5 spinal nerves (C5-T1), from approximately $5 \mathrm{~mm}$ distal to their exit from the intervertebral foramina, to proximal $1 \mathrm{~cm}$ of distal branches. All spinal nerves were tagged on the cranial aspect of their circumference using 10-0 nylon suture for orientation. The fascicular dissection of the C5-T1 spinal nerves was performed under microscopic magnification. The area occupied by different nerve fascicles was then expressed as a percentage of the total cross-sectional area of a spinal nerve.

RESULTS The localization of fascicular groups was fairly consistent in all spinal nerves. Overall, $4 \%$ of the plexus supplies the suprascapular nerve, $31 \%$ supplies the medial cord (comprising the ulnar nerve and medial root of the median nerve [MN]), 27.2\% supplies the lateral cord (comprising the musculocutaneous nerve and lateral root of the MN), and $37.8 \%$ supplies the posterior cord (comprising the axillary and radial nerves).

CONCLUSIONS The fascicular dissection and definitive anatomical localization of fascicular groups is feasible in plexal spinal nerves. The knowledge of exact fascicular location might be translatable to the operating room and can be used to anastomose related fascicles in brachial plexus surgery, thereby avoiding the possibility of axonal misrouting and improving the results of plexal reconstruction.

http://thejns.org/doi/abs/10.3171/2015.6.JNS142181

KEY WORDS brachial plexus; cadaver study; fascicular topography; peripheral nerve

$\mathrm{T}$ RAUMATIC brachial plexus injuries are devastating in nature as they primarily affect young individuals during the most productive years of their lives. ${ }^{1}$ There has been a significant development in the management of these injuries over the last decades, especially with the advent of microsurgical techniques, and use of magnification, microsutures, and microinstruments. ${ }^{26}$ Many advances have been made in the areas of neurobiology of nerve injury and regeneration, and increasing attempts are being made in the use of nerve allografts and nerve conduits for bridging the gaps., ${ }^{2,13,14,16}$ Recently introduced nerve transfer techniques selectively neurotize target muscles close to motor endplates and produce early and consistently good results, especially in the management of upper truncal injuries. ${ }^{3,15,21,32}$
The management of brachial plexus injuries involves a combination of comprehensive care, cross-specialty consultations, prolonged hours of surgery, and a protracted period of pre- and postoperative physiotherapy. Even then, the results of surgery may not be favorable. ${ }^{25}$ Various modalities have been used in the surgical treatment of these devastating injuries, depending on the type of injury. These modalities consist of either neurolysis, or intra/extraplexal nerve transfer (neurotization), with or without a nerve graft. ${ }^{3,11,12,26,27}$ Two factors play a major role in functional recovery after the repair of a nerve lesion. ${ }^{24}$ The first factor is the number of axons that successfully cross the anastomotic site. On average, approximately $30 \%$ of the axons are lost while traveling across one anastomotic site. However, attempts to prevent the axonal loss across the anastomotic

ABBREVIATIONS $A N=$ axillary nerve; $L C=$ lateral cord; $L P N=$ lateral pectoral nerve; $L R M N=$ lateral root of the $M N ; M C=$ medial cord; $M C N=$ musculocutaneous nerve; $M C N A=$ medial cutaneous nerve of the arm; $M C N F=$ medial cutaneous nerve of the forearm; $M N=$ median nerve; $M R M N=$ medial root of the MN; $P C=$ posterior cord; $\mathrm{RN}=$ radial nerve; $\mathrm{SSN}=$ suprascapular nerve; $\mathrm{UN}=$ ulnar nerve.

SUBMITTED September 20, 2014. ACCEPTED June 9, 2015.

INCLUDE WHEN CITING Published online December 11, 2015; DOI: 10.3171/2015.6.JNS142181. 
site have not been successful in clinical practice thus far, despite several efforts. ${ }^{31}$ The reestablishment of original fascicular connections by outgrowing axons is the second and perhaps the most important factor in determining surgical outcome. Among these factors, the surgeon can influence the routing of axons by connecting related fascicles in the proximal and distal stumps..$^{24}$ The misalignment of axons after the presently popular techniques of nerve transfer and neurotization is a distinct possibility, which has a significant negative bearing on the surgical outcome after nerve repairs. Henceforth, fascicular nerve repairs should produce better results than epineurial repairs. The fascicular nerve repair might still offer the best solution for nerve recovery, as fascicular microdissection techniques have improved with the advent of the operating microscopes.

The aim of this cadaveric study was to study the fascicular topography of the brachial plexus in the spinal nerves. This fascicular mapping could provide the nerve surgeon with the knowledge of fascicular orientation in the spinal nerves of the brachial plexus. This knowledge might improve the results of nerve grafting in brachial plexus injuries, by anastomosing the related fascicles and avoiding axonal misrouting.

\section{Methods}

\section{Preparation of the Dissection Sample}

We received institutional ethical committee clearance before beginning the study. The study was performed on the right-sided brachial plexus of 25 fresh adult male cadavers. The cadavers were placed supine and the entire supraclavicular and infraclavicular portion of the plexus was dissected in a standard manner. The C5-T1 spinal nerves were identified and tagged on their cranial aspects (from the nerve surgeon's perspective) using a 10-0 nylon suture, approximately $5 \mathrm{~mm}$ distal to their exit from the intervertebral foramen. The ventral aspect of the C-5 spinal nerve was defined from the nerve surgeon's perspective, as the point where it is crossed by the phrenic nerve.

The infraclavicular exposure was then conducted in a standard manner. The divisions, cords, and branches were then dissected in a usual manner. The clavicle was then cut to facilitate the transection of the complete brachial plexus from the level of tagged spinal nerves to cords, along with a proximal $1 \mathrm{~cm}$ of all the distal plexal branches (Fig. 1). The specimen was then immersed immediately in a fixation solution comprising $10 \%$ formaldehyde, phenolic acid, and water for at least 48-72 hours.

The fixed specimen was then dissected under magnification of an operating microscope (Carl Zeiss). The orientation in space was maintained with the help of the 10-0 nylon tagging suture on the cranial aspect of the spinal nerve. The fascicular dissection of the C5-T1 spinal nerves was then performed using microsurgical techniques, with meticulous removal of the overlying fascia and epineurium and subsequent identification and tracing of nerve fascicles from individual distal branches to the proximal spinal nerve.

\section{Calculation of the Fascicular Topography}

The roots were sectioned transversely at a proximal level, and a manual sketch of the circumference of the spinal nerve at each level was constructed. The picture of the transverse section at each level was then divided into 4 quadrants (rostroventral, caudoventral, rostrodorsal, and caudodorsal), keeping the nylon stitches as a marker at the 12 o'clock position (in reference to the hands of a clock). The area occupied by different nerve fascicles was then manually defined and also expressed as a percentage of the total cross-sectional area of the spinal nerve. The anatomical position of the nerve fascicles inside all the spinal nerves was thus assessed and registered manually. The percentage area occupied by each fascicle is reported as the mean value across all the specimens.

\section{Results}

The contribution of the various fascicles to the brachial plexus and their spatial locations in the C5-T1 spinal nerves is illustrated in Fig. 2.

\section{C-5 Spinal Nerve}

The C-5 spinal nerve consisted of fascicles of the suprascapular nerve (SSN), musculocutaneous nerve (MCN), lateral cord (LC) contribution of the anterior division, posterior cord (PC), and the axillary nerve (AN; Fig. 3A). The SSN fascicle was monofascicular in nature, originating solely from the C-5 root in all specimens. The mean cross-sectional area of the C-5 root occupied by the SSN fascicles was $20 \%$, and the location of these fascicles was in the rostroventral quadrant, from the 12 o'clock to the 2 o'clock position. The fascicles going to the LC occupied $30 \%$ of the area of the C-5 spinal nerve in the ventrocaudal quadrant. The MCN fascicles were found in the most ventral aspect of this quadrant, occupying $15 \%$ of the total cross-sectional area, which was half of the area occupied by the LC fascicles. The whole of the PC fascicles was located in the dorsal aspect of the spinal nerve, with the AN fascicles occupying $20 \%$ of the area in the rostrodorsal quadrant, and the rest of the PC occupying $30 \%$ of the area in the dorsocaudal quadrant.

\section{C-6 Spinal Nerve}

The C-6 spinal nerve consisted of the fascicles of the $\mathrm{MCN}$, lateral pectoral nerve (LPN), lateral root of the median nerve (LRMN), radial nerve (RN), and AN (Fig. 3B). The majority (40\%) of the cross-sectional area of C- 6 was occupied by MCN fascicles, located in the ventral quadrant, from the 12 o'clock to the 6 o'clock position. The LPN and the LRMN occupied $8 \%$ of the area, each, in the ventrocaudal and dorsocaudal quadrants, respectively. The RN fascicles occupied the dorsal quadrant, with the AN fascicles located in the rostral-most part of the dorsal quadrant and occupying $12 \%$ of the area of the root. The rest of the dorsal quadrant was occupied by the RN fascicles, covering $32 \%$ of the area of the spinal nerve. The individual branches of the PC, except the RN, were not mapped as they occupied a very small area of the C-6 root, and it was not possible to quantify their area and location.

\section{C-7 Spinal Nerve}

The C-7 spinal nerve consisted of the fascicles of 


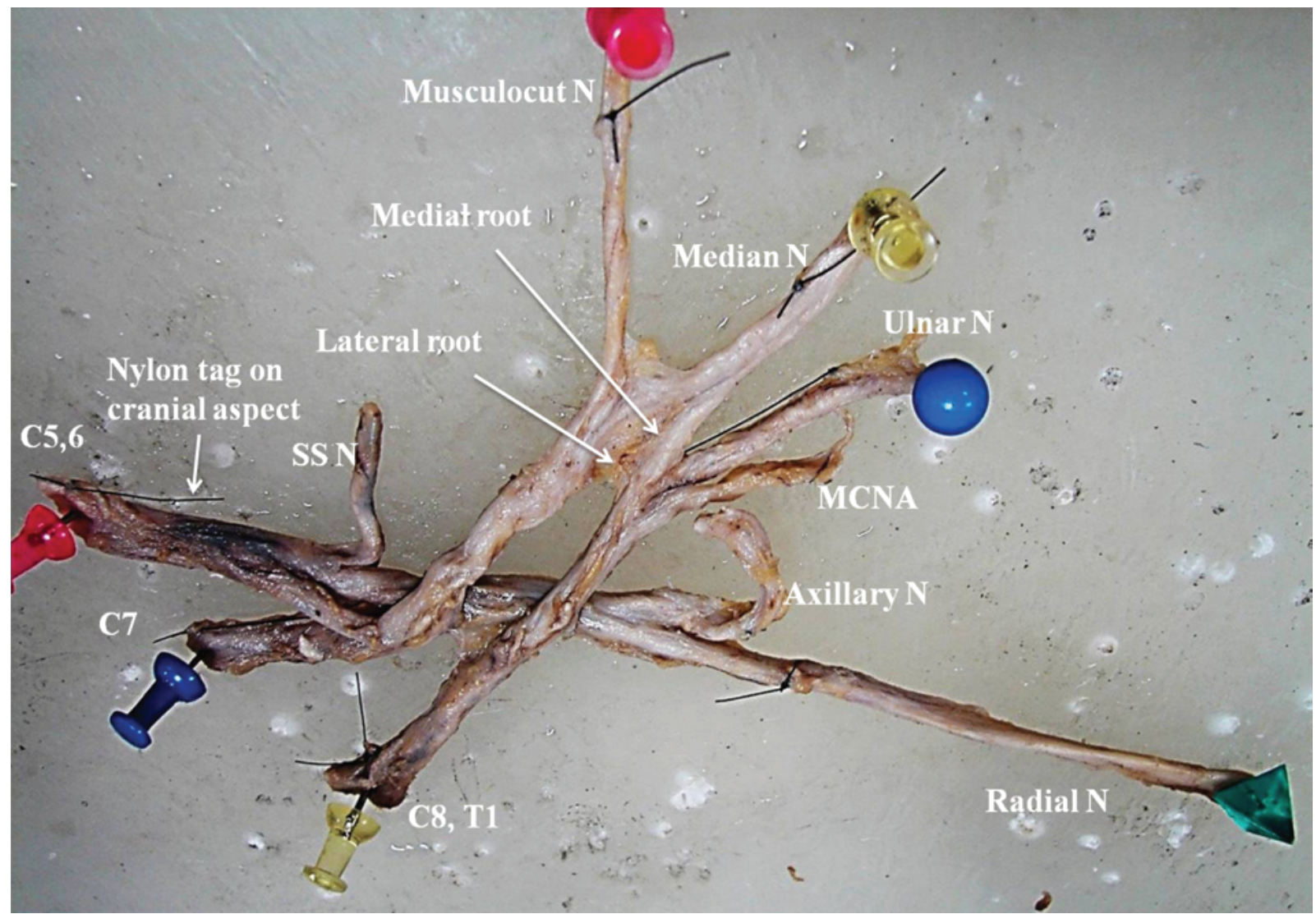

FIG. 1. Photograph of a labeled, preserved specimen of the right-sided brachial plexus. Musculocut = musculocutaneous; $N=$ nerve. Figure is available in color online only.

LRMN, LPN, ulnar nerve (UN), and RN (Fig. 3C). The fascicles to the LRMN were found in most of the ventral half of the C-7 root, occupying $40 \%$ of the cross-sectional area. The rest of the $10 \%$ of the ventral half was occupied by the LPN fascicles, lying in the ventrocaudal quadrant. The RN and UN fascicles were found in the dorsal half of C-7, occupying $25 \%$ each of this area, respectively. The RN fascicles were located from the 9 o'clock to the 12 o'clock position, and the UN fascicles between the 6 o'clock to the 9 o'clock positions dorsally.

\section{C-8 Spinal Nerve}

The whole of the ventral half $(50 \%)$ of the C-8 spinal nerve, from the 12 o'clock to the 6 o'clock position, was occupied with the fascicles going to the medial root of the MN (MRMN; Fig. 3D). The rest of the dorsal half contained the fascicles of the UN, PC, medial cutaneous nerve of the arm (MCNA), and medial cutaneous nerve of the forearm (MCNF). The PC fascicles occupied the rostrodorsal quadrant and extended to the dorsocaudal quadrant, occupying $30 \%$ of the cross-sectional area of C-8. The UN fascicles occupied $15 \%$ of the area, and the MCNA/MCNF combined occupied 5\% of the area, in the dorsocaudal quadrant.

\section{T-1 Spinal Nerve}

The T-1 spinal nerve consisted of fascicles of the medial cord (MC) on the ventral aspect from the 12 o'clock to the 5 o'clock position, covering $30 \%$ of the cross-sectional area (Fig. 3E). The UN fascicles were sandwiched between the PC fascicles on the dorsal aspect, occupying $30 \%$ of the area from the 7 o'clock to the 10 o'clock position. The rest of the area was occupied by the PC fascicles, covering $40 \%$ of the area of the root, in the rostrodorsal and dorsocaudal quadrants.

The fascicles to the smaller branches of the brachial plexus, such as nerve to subclavius, nerve to rhomboids, thoracodorsal nerve, medial pectoral nerve, and subscapular nerves, were not identifiable on these 25 specimens as they had a very small contribution. The contribution of these nerve fascicles to the brachial plexus was therefore not localized or quantified. The PC mainly consisted of the RN fascicles, as the other nerve fascicles could not be isolated because of reasons mentioned above.

The area occupied by the respective nerve branches in the brachial plexus is summarized in Table 1 . It could be summarized from this study that approximately $4 \%$ of the brachial plexus supplies the SSN, 31\% supplies the MC (comprising the UN and MRMN), 27.2\% supplies the lateral cord (comprising the MCN and LRMN), and 37.8\% supplies the PC (comprising the AN and RN).

\section{Discussion}

Brachial plexus injuries are the most severe nerve injuries of the upper extremity, resulting in marked functional impairment and the severe morbidity caused by these le- 

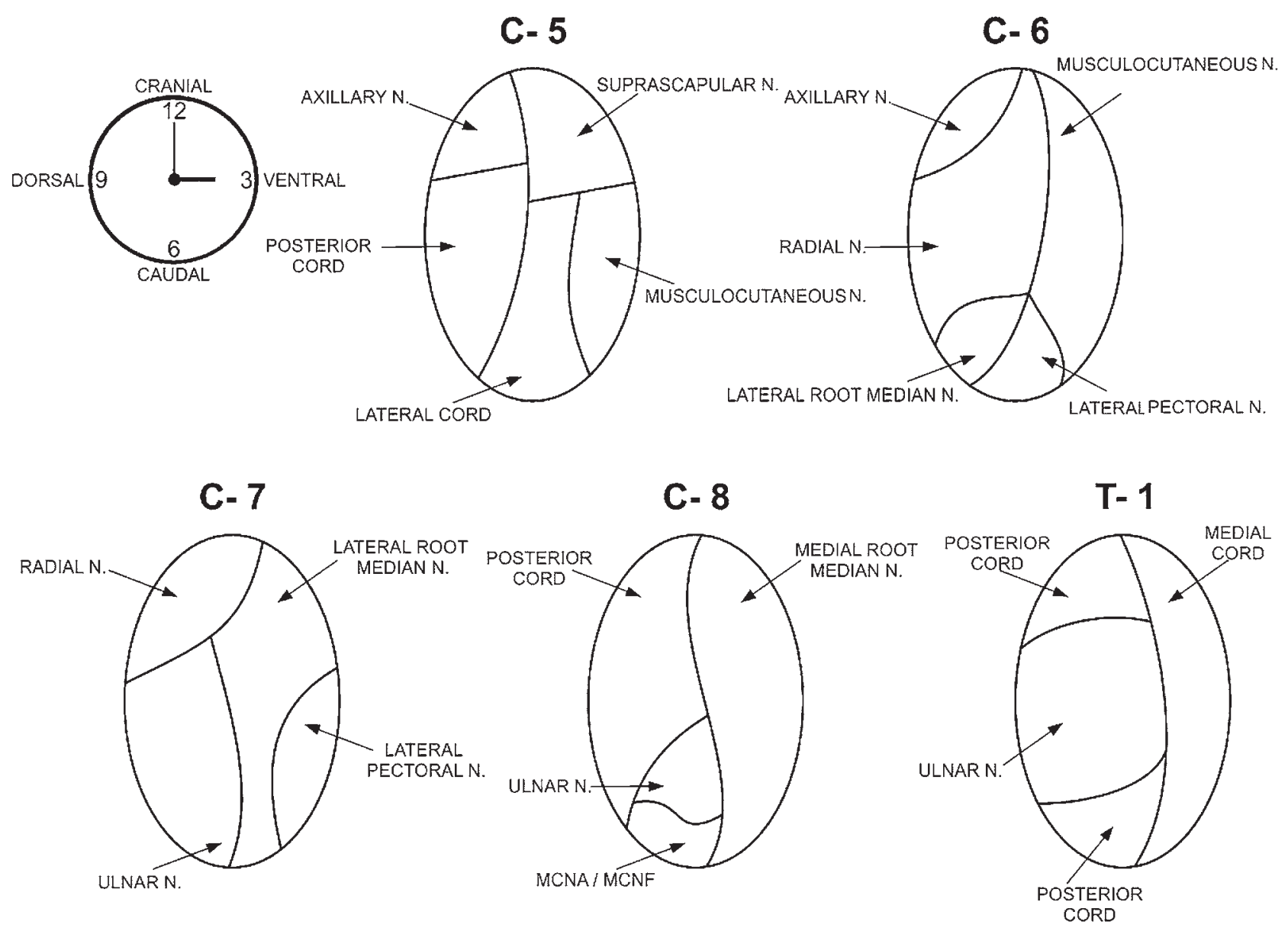

FIG. 2. Illustration showing the spatial localization of fascicular groups in the C5-T1 spinal nerves.

sions. ${ }^{11,17}$ The results of brachial plexus injury have been quite dismal in the past, but multiple studies have documented consistently good results after surgery in recent years due to the availability of good quality suture materials and the advent of the operating microscope. ${ }^{11,18,20,26}$ The success rate for surgery in brachial plexus injuries ranges from $25 \%$ to $85 \%$, depending upon whether they are partial- or pan-plexal injuries. ${ }^{3,12,20,27}$

Surgery is indicated in cases of the absence of signs of regeneration, even after 3-4 months of injury. ${ }^{11,13}$ There is no standard treatment strategy that suits all patients, and an individualistic approach is preferred in the treatment of these lesions. Although better results are being achieved in recent times, they are not up to the expected standards. ${ }^{24,26}$ This might be ascribed to the misrouting of nerve fascicles during surgery.

The gross anatomy of the brachial plexus appears fairly consistent and straightforward; however, frequent variations occur among individuals with respect to the contribution of the roots to the plexus and the relative position of the individual fascicles in the constituents of the plexus..$^{10,22,23}$ For this purpose, a complete topographical evaluation and mapping of the brachial plexus would aid the brachial plexus surgeon in the surgical reconstruction of the plexus in a manner that might potentially improve results.
To date, very few studies ${ }^{8,19,24,37}$ have been reported in the literature that focused on the fascicular microanatomy of the brachial plexus, although a few researchers have mapped the individual nerves of the brachial plexus in-

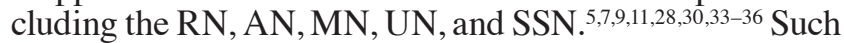
mapping is important because it provides a precise knowledge of the orientation of individual fascicles in the nerve roots so that nerve repair with or without graft or nerve transfers could be successfully performed.

The first type of this kind of study was published by Sunderland in 1945, in which the internal anatomy of the $\mathrm{RN}, \mathrm{MN}$, and UN was described. ${ }^{28}$ Another report was published in 1959 by the same author in which he mapped the AN and MCN..$^{30}$ It should be noted that in both of these studies, only the individual nerves in the arm and forearm were dissected from the level of the cords of the brachial plexus (lateral, posterior, and medial cords) and distally. These studies offered an impetus for other researchers to work further in this aspect. The present study is an attempt to map the individual fascicles in the individual spinal nerves of the brachial plexus immediately after their exit from the intervertebral neural foramen.

Yokoyama $^{37}$ studied the intraneural topography of the brachial plexus in 10 Japanese adult cadavers and concluded that in the C-5 root, the SSN fasciculus was located cranially, MCN ventrally, and AN posteriorly. Although 

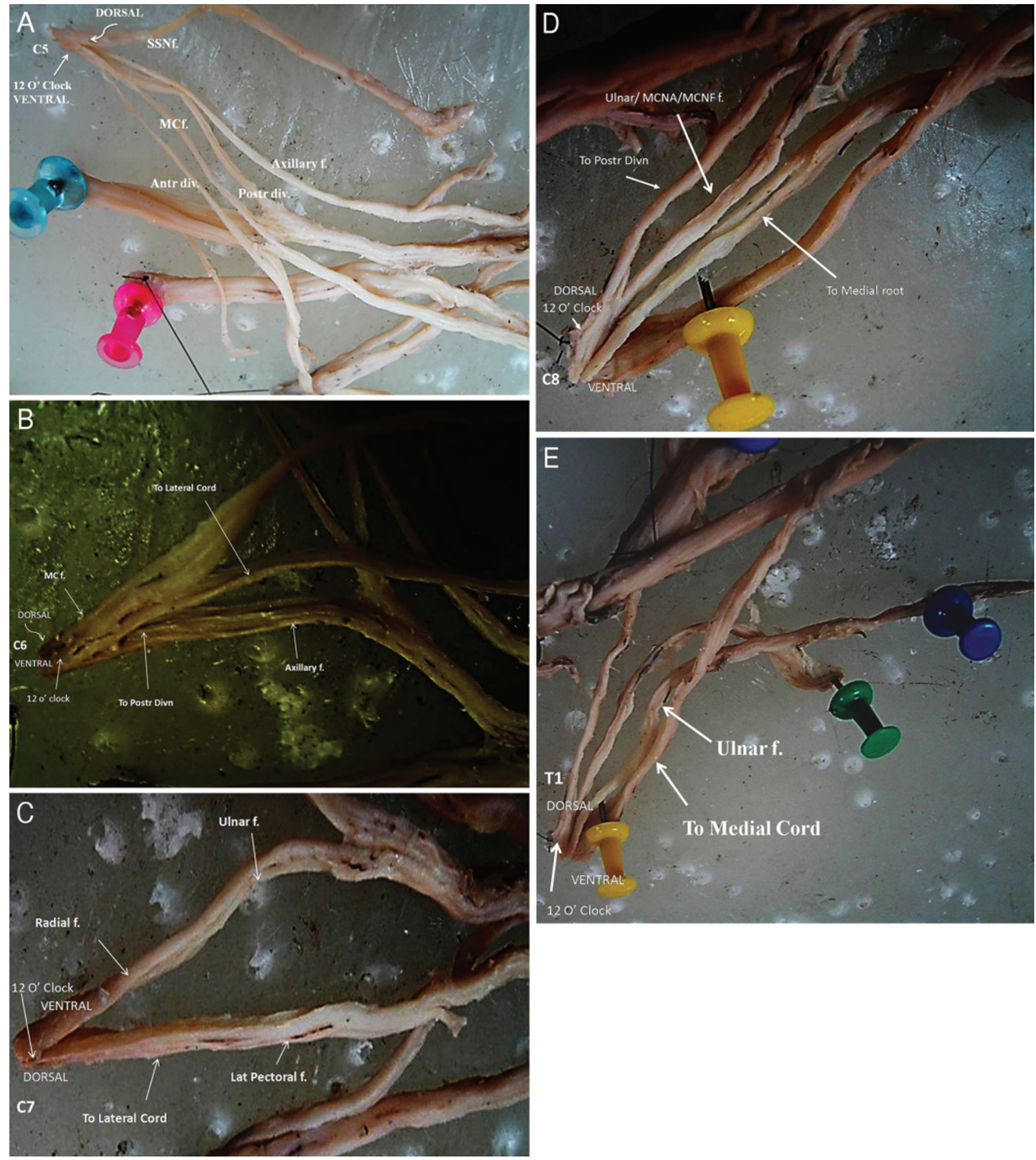

FIG. 3. Operative microscope photographs showing localization of individual fascicles in C5-T1 spinal nerves: C-5 (A), C-6 (B), C-7 (C), C-8 (D), T-1 (E). antr = anterior, $\operatorname{div}(\mathrm{n})=\operatorname{division}, \mathrm{f}=$ fascicle, lat pectoral = lateral pectoral, $\mathrm{MC}=$ musculocutaneous, postr $=$ posterior. Figure is available in color online only.

the authors mentioned the fact that the intraneural location of fascicles from the cord level to the distal level of nerve roots could be identified, no such mention about the topography of other nerve roots could be found in their results, as this article was published in Japanese. Another similar study on intraneural topography concluded that the SSN fascicle was located cranially in the C-5 root. ${ }^{23}$ In another Japanese report on 4 brachial plexus dissection 
TABLE 1. Contributions of various nerves to the brachial plexus

\begin{tabular}{lcc}
\hline \multicolumn{1}{c}{ Nerves } & \% Contribution to Brachial Plexus & Major Contributing Root (\% area occupied by respective fascicles) \\
\hline SSN & 4 & C-5 $(20 \%)$ \\
\hline Axillary & 6.4 & C-5 $(20 \%)+$ C-6 $(12 \%)$ \\
\hline MCN & 11 & C-5 $(15 \%)+$ C-6 $(40 \%)$ \\
\hline PC (AN + RN) & 37.8 & C-5 $(50 \%)+$ C-6 $(44 \%)+$ C-7 $(25 \%)+$ C-8 $(30 \%)+$ T-1 $(40 \%)$ \\
\hline MC (UN + MRMN) & 31 & C-7 $(25 \%)+$ C-8 $(70 \%)+$ T-1 $(60 \%)$ \\
\hline LC (MCN + LRMN) & 27.2 & C-5 $(30 \%)+$ C-6 $(56 \%)+$ C-7 $(50 \%)$ \\
\hline
\end{tabular}

samples, the authors concluded that there are considerable variations in the constituent elements of the brachial plexus and that nerve fascicles were most easily discernible at the cord level of the plexus. They also indicated that nerve reconstructive procedures could be performed with success at the cord level or at the division level. ${ }^{8}$

Previously, the internal topography of the major nerves of the forearm and hand such as the MN and UN have been described separately by Williams and Jabaley ${ }^{35}$ and Jabaley et al. ${ }^{9}$ They studied the complex interfascicular relationships between the various nerves and their branching patterns and described their importance in nerve repair surgeries. Jabalay et al..$^{9}$ also concluded that fascicles of the MN and UN could be separated more easily and did not branch over long distances.

Siqueira et al. ${ }^{24}$ analyzed the topography of the C-5 nerve root in 20 adult cadaveric specimens specifically looking for the SSN contribution and its precise orientation in the C-5 root. They concluded that the SSN is monofascicular and occupied approximately $28 \%$ of the C-5 root. The authors also reported that the SSN was located in the rostroventral quadrant (between the 9 o'clock and 3 o'clock positions) in $80 \%$ of cases and coaptation in this region would probably reduce axonal misrouting and improve outcome.

We studied the fascicular topography of C5-T1 plexal nerve roots individually in 25 adult male cadavers. Only the right-sided plexal roots were studied to maintain homogeneity. Three important characteristics were assessed: localization of fascicular groups in each spinal nerve (in relation to quadrants), average cross-sectional contribution of these nerve fascicles in each spinal nerve, and the proportion of the brachial plexus contributing to the major nerves of the upper limb.

In our study, the SSN also occupied the ventral compartment and was monofascicular in nature. The SSN occupied a narrow range of the cross-sectional area of the C-5 spinal nerve from the 12 o'clock to 2 o'clock position in the rostroventral quadrant, as compared with the much wider area reported by Siqueira et al. (9 o'clock to 3 o'clock position). ${ }^{16}$ Another important finding was that the location of the SSN was constant in all our specimens, in contrast to the study of Siqueira et al. in which they found such a localization in only $80 \%$ of cases. ${ }^{16}$ Thus, we could clearly establish the localization of the SSN within this narrow range in the rostroventral compartment, such that coaptation of the nerve graft to the SSN fascicle in this area of the C-5 nerve would theoretically contribute to better results.
Also, the location of the other nerve fascicles in the C-5 spinal nerve, such as the MCN and AN, were similar to that reported by Yokoyama, thus reconfirming his findings. ${ }^{37}$ With respect to the topography of the remaining plexal roots, no other studies could be found in the literature, and to the best of the authors' knowledge, ours is the first type of such a study to be reported. The major contributions in each of the spinal nerve roots were as follows: AN for C-5 (20\%), MCN for C-6 (40\%), LRMN for C-7 (40\%), MRMN for C-8 (50\%), and UN for C-8 and T-1 roots $(45 \%)$ combined. The contribution for the $\mathrm{RN}$ originated uniformly from all the roots in our study $(30 \%$, $32 \%, 25 \%, 30 \%$, and $40 \%$ for C5-T1 spinal nerves, respectively). In an electrophysiological study by $\mathrm{Gu}$ et al., ${ }^{6}$ the functional motor innervation of the normal brachial plexus roots (during contralateral C-7 transfer) showed results very similar to those in our study, except in the contribution to the RN. The authors noted that C-5 mainly forms the AN, C-6 mainly the MCN, C-7 mainly the RN, C-8 mainly the MN, and T-1 mainly forms the UN. However, in their study, only the representative muscles were sampled electrophysiologically, for example, triceps and extensor digitorum communis in the case of the RN. The RN contribution of the T- 1 spinal nerve (40\%) in our study could be due to these fascicles supplying other muscles of the wrist and hand.

Another important finding of our study was the percentage of the brachial plexus contributing to the major nerves of the upper limb. It was found that $4 \%$ of the plexus supplies the SSN, and the contribution of brachial plexus to the PC, MC, and LC was $37.8 \%, 31 \%$, and $27.2 \%$, respectively (Table 1). It was not possible to identify the exact contribution of the plexus to the UN and MN because it was difficult to follow the fascicles of these nerves in every specimen.

Although, theoretically, fascicular repair would appear to have better success than epineural repair, the debate still exists among various researchers and clinicians, with some authors reporting excellent functional outcomes in nerve transfers or grafting, not necessarily due to original interfascicular reconnections..$^{29,38}$ Although not proven in humans, a study on rat sciatic nerves by Brushart et al. in 1983 confirmed the superiority of fascicular repair versus epineural repair. ${ }^{4}$ Although it is believed that fascicular repair is appropriate in distal nerves, it is not yet practiced in the proximal plexus at the root level, probably because of the difficulty in accomplishing proper alignment due to hematoma, swelling, or scar tissue. This study highlights the possibility of fascicular dissection in all the plexal 
roots and fascicular orientation knowledge, which could be translated to the operating theater for future nerve reconstructive surgeries.

The limitation of the study is its small sample size. Furthermore, the area occupied by the respective fascicles of the plexus is only a manual estimate and not an exact measure. It is for these reasons that we could not comment on the anatomical variations from one brachial plexus to another.

\section{Conclusions}

Fascicular microdissection is possible with careful dissection. Definitive anatomical localization of fascicular groups is feasible in plexal elements, and localization of fascicles was consistent in all samples. The SSN is monofascicular and occupies the rostroventral quadrant of the C-5 root. Four percent of the plexus supplies the SSN, and the contribution of the brachial plexus to the PC, MC, and LC was $37.8 \%, 31 \%$, and $27.2 \%$, respectively. The exact fascicular location is translatable to the operating room, and this knowledge can be used to anastomose related fascicles in brachial plexus surgery, thereby avoiding axonal misrouting and possibly improving the results of nerve grafting.

\section{Acknowledgments}

The study was supported by a research grant from the Indian Council of Medical Research, New Delhi, India. We thank Dr. Sanjeev Lalwani, Additional Professor, Department of Forensic Medicine, All India Institute of Medical Sciences, New Delhi, who provided technical support by providing fresh, soft, embalmed cadavers for the study.

\section{References}

1. Aszmann OC, Dellon AL: The internal topography of the axillary nerve: an anatomic and histologic study as it relates to microsurgery. J Reconstr Microsurg 12:359-363, 1996

2. Belkas J, Shoichet MS, Midha R: Axonal guidance channels in peripheral nerve regeneration. Oper Tech Orthop 14:190-198, 2005

3. Bhandari PS, Sadhotra LP, Bhargava P, Bath AS, Mukherjee MK, Bhatti T, et al: Surgical outcomes following nerve transfers in upper brachial plexus injuries. Indian J Plast Surg 42:150-160, 2009

4. Brushart TM, Tarlov EC, Mesulam MM: Specificity of muscle reinnervation after epineurial and individual fascicular suture of the rat sciatic nerve. J Hand Surg Am 8:248-253, 1983

5. Chow JA, Van Beek AL, Meyer DL, Johnson MC: Surgical significance of the motor fascicular group of the ulnar nerve in the forearm. J Hand Surg Am 10:867-872, 1985

6. Gu Y : Functional motor innervation of brachial plexus roots. An intraoperative electrophysiological study. Chin Med J (Engl) 109:749-751, 1996

7. Hasegawa O, Ohta S, Komiyama A, Kurita R, Nara Y: [Intraneural topography of the median nerve at the elbow level-an analysis using intraneural neurography.] No To Shinkei 47:147-151, 1995 (Jpn)

8. Honda K: [Study on the intraneural topography of the brachial plexus.] Nippon Seikeigeka Gakkai Zasshi 67:58-70, 1993 (Jpn)

9. Jabaley ME, Wallace WH, Heckler FR: Internal topography of major nerves of the forearm and hand: a current view. $\mathbf{J}$ Hand Surg Am 5:1-18, 1980

10. Johnson EO, Vekris M, Demesticha T, Soucacos PN: Neuro- anatomy of the brachial plexus: normal and variant anatomy of its formation. Surg Radiol Anat 32:291-297, 2010

11. Kandenwein JA, Kretschmer T, Engelhardt M, Richter HP, Antoniadis G: Surgical interventions for traumatic lesions of the brachial plexus: a retrospective study of 134 cases. $\mathbf{J}$ Neurosurg 103:614-621, 2005

12. Kim DH, Cho YJ, Tiel RL, Kline DG: Outcomes of surgery in 1019 brachial plexus lesions treated at Louisiana State University Health Sciences Center. J Neurosurg 98:1005-1016, 2003

13. Krekoski CA, Neubauer D, Zuo J, Muir D: Axonal regeneration into acellular nerve grafts is enhanced by degradation of chondroitin sulfate proteoglycan. J Neurosci 21:6206-6213, 2001

14. Mackinnon SE, Dellon AL: Clinical nerve reconstruction with a bioabsorbable polyglycolic acid tube. Plast Reconstr Surg 85:419-424, 1990

15. Merrell GA, Barrie KA, Katz DL, Wolfe SW: Results of nerve transfer techniques for restoration of shoulder and elbow function in the context of a meta-analysis of the English literature. J Hand Surg Am 26:303-314, 2001

16. Midha R: Emerging techniques for nerve repair: nerve transfers and nerve guidance tubes. Clin Neurosurg 53:185-190, 2006

17. Midha R: Epidemiology of brachial plexus injuries in a multitrauma population. Neurosurgery 40:1182-1188, discussion 1188-1189, 1997

18. Millesi H: Surgical management of brachial plexus injuries. J Hand Surg Am 2:367-378, 1977

19. Narakas A: [Indications and results of direct surgical treatment in lesions caused by stretching of the brachial plexus. I. Indications for direct surgical treatment.] Rev Chir Orthop Appar Mot 63:88-106, 1977 (Fr)

20. Narakas A: Surgical treatment of traction injuries of the brachial plexus. Clin Orthop Relat Res (133):71-90, 1978

21. Oberlin C, Béal D, Leechavengvongs S, Salon A, Dauge MC, Sarcy JJ: Nerve transfer to biceps muscle using a part of ulnar nerve for C5-C6 avulsion of the brachial plexus: anatomical study and report of four cases. J Hand Surg Am 19:232-237, 1994

22. Ongoïba N, Destrieux C, Koumare AK: [Anatomical variations of the brachial plexus.] Morphologie 86:31-34, 2002 (Fr)

23. Pellerin M, Kimball Z, Tubbs RS, Nguyen S, Matusz P, Cohen-Gadol AA, et al: The prefixed and postfixed brachial plexus: a review with surgical implications. Surg Radiol Anat 32:251-260, 2010

24. Siqueira MG, Foroni LHL, Martins RS, Chadi G, Malessy MJ: Fascicular topography of the suprascapular nerve in the C5 root and upper trunk of the brachial plexus: a microanatomic study from a nerve surgeon's perspective. Neurosurgery 67 (2 Suppl Operative):402-406, 2010

25. Siqueira MG, Martins RS: Surgical treatment of adult traumatic brachial plexus injuries: an overview. Arq Neuropsiquiatr 69:528-535, 2011

26. Songcharoen P: Management of brachial plexus injury in adults. Scand J Surg 97:317-323, 2008

27. Sulaiman OA, Kim DD, Burkett C, Kline DG: Nerve transfer surgery for adult brachial plexus injury: a 10-year experience at Louisiana State University. Neurosurgery 65 (4 Suppl):A55-A62, 2009

28. Sunderland S: The intraneural topography of the radial, median and ulnar nerves. Brain 68:243-299, 1945

29. Sunderland S: The pros and cons of funicular nerve repair. J Hand Surg Br 4:201, 1979

30. Sunderland S, Marshall RD, Swaney WE: The intraneural topography of the circumflex, musculocutaneous and obturator nerves. Brain 82:116-129, 1959

31. Tannemaat MR, Boer GJ, Eggers R, Malessy MJ, Verhaagen 
J: From microsurgery to nanosurgery: how viral vectors may help repair the peripheral nerve. Prog Brain Res 175:173186,2009

32. Venkatramani H, Bhardwaj P, Faruquee SR, Sabapathy SR: Functional outcome of nerve transfer for restoration of shoulder and elbow function in upper brachial plexus injury. J Brachial Plex Peripher Nerve Inj 3:15, 2008

33. Watchmaker GP, Gumucio CA, Crandall RE, Vannier MA, Weeks PM: Fascicular topography of the median nerve: a computer based study to identify branching patterns. J Hand Surg Am 16:53-59, 1991

34. Watchmaker GP, Lee G, Mackinnon SE: Intraneural topography of the ulnar nerve in the cubital tunnel facilitates anterior transposition. J Hand Surg Am 19:915-922, 1994

35. Williams HB, Jabaley ME: The importance of internal anatomy of the peripheral nerves to nerve repair in the forearm and hand. Hand Clin 2:689-707, 1986

36. Yang LJ, Chang KW, Chung KC: A systematic review of nerve transfer and nerve repair for the treatment of adult upper brachial plexus injury. Neurosurgery 71:417-429, 2012

37. Yokoyama I: [Study on the intraneural topography of the brachial plexus.] Nippon Seikeigeka Gakkai Zasshi 63:10851102,1989 (Jpn)

38. Young L, Wray RC, Weeks PM: A randomized prospective comparison of fascicular and epineural digital nerve repairs. Plast Reconstr Surg 68:89-93, 1981

\section{Disclosures}

The authors report no conflict of interest concerning the materials or methods used in this study or the findings specified in this paper.

\section{Author Contributions}

Conception and design: Sinha. Acquisition of data: Sinha, Prasad. Drafting the article: Prasad. Critically revising the article: Sinha. Approved the final version of the manuscript on behalf of all authors: Sinha. Administrative/technical/material support: Lalwani.

\section{Supplemental Information}

Previous Presentations

Portions of this work were presented at the 2012 AANS annual meeting in Miami, Florida. Portions of this work were also presented and awarded the best poster award at the 61st Neurological Society of India annual meeting in New Delhi, December 19-21, 2012.

\section{Correspondence}

Sumit Sinha, Department of Neurosurgery, Jai Prakash Narayan Apex Trauma Centre, AIIMS, Rm. 307, 3rd Fl., F 25 Ansari Nagar, New Delhi 110069, India. email: sumitaiims@yahoo.com. 